\title{
Ajuste académico y bienestar psicológico en estudiantes de la universidad pública de Guatemala
}

Academic adjustment and psychological well-being in students at the Public University of Guatemala

Donald W. González-Aguilar ${ }^{1}$ https://doi.org/10.46954/librosfahusac.6

\section{Resumen}

OBJETIVO: determinar la asociación del bienestar psicológico en ajuste académico de los estudiantes de la universidad pública de Guatemala. MÉTODo: el enfoque del estudio fue cuantitativo, no experimental, de corte transversal y alcance correlacional predictivo. Se utilizó la escala de bienestar psicológico de Ryff y la Medida de Participación Escolar. RESULTADOS: se evaluaron 347 estudiantes de la universidad pública de Guatemala. La edad $M=22(S D=2.09), 228$ (65.70\%) mujeres. La razón de posibilidades $(O R)$ para un indicador alto de bienestar psicológico fue de 1.511 (IC 95\% 2.881 - 7.131) asociado al ajuste académico, con una varianza explicada de $16.50 \%$ (Nagelkerke $R^{2}$ ). El modelo fue significativo $(p<.001)$. 
CONCLUSIÓN: quienes tienen indicadores altos de bienestar psicológico y ajuste académico tienden a involucrarse en su universidad, en actividades positivas, dedican su tiempo libre a desarrollar su potencial académico y su motivación para participar en actividades dentro o fuera del aula. Además, mejorarán su capacidad, competencia y valores, ayudándolos a transitar de mejor forma a la vida profesional.

Palabras clave: participación escolar, bienestar, universidad, clima escolar.

\section{Abstract}

OBJECTIVE: to determine the association between psychological well-being and academic adjustment of students at the Public University of Guatemala. METHOD: the approaches of this study was quantitative, nonexperimental, cross-sectional, and predictive correlational scope. Using the Ryff psychological well-being scale and the School Participation Measure were used. RESULTS: 347 students from a public university in Guatemala were evaluated. The age $M=22$ ( $S D=2.09), 228(65.70 \%)$ women. The odds ratio $(O R)$ for a high indicator of psychological well-being was 1.511 (95\% CI 2.881 - 7.131) associated with academic adjustment, with an explained variance of $16.50 \%\left(\right.$ Nagelkerke $\left.R^{2}\right)$. The model was significant $(p<.001)$. CONCLUSION: students who have high indicators of psychological well-being and academic adjustment tend to be involved in their university, engaging in positive activities, dedicating their free time to develop their academic potential and their motivation to participate in activities inside or outside the classroom. In addition, they will improve their ability, competence, and values, helping them to correctly pass their transition stage to professional life.

Keywords: school participation, wellness, university, school climate. 


\section{Introducción}

En las últimas décadas, ha incrementado el interés en el bienestar de los estudiantes a nivel de políticas educativas, y esta atención llevó a la investigación a centrarse en el estudio de los aspectos psicológicos relacionados con los procesos de aprendizaje. En este sentido, varias investigaciones tenían como objetivo descubrir si los sistemas educativos podrían contribuir al bienestar de los estudiantes y de qué manera. (Seligman, 2011).

Particularmente la universidad donde se hace la investigación es institución de educación formal financiada por el gobierno. Para Santrock (2009), la educación superior funciona no solo para el aprendizaje sino también para desarrollar la moral, el carácter, la aptitud y el interés de los estudiantes. Es un medio potencial para desarrollar la personalidad del individuo, que afecta el desarrollo de los estudiantes en alguno o varios aspectos de la vida como la propia identidad; creencia en la capacidad, descripción de la vida, relación interpersonal, restricción de normas entre lo bueno y lo malo y el concepto de sistema social al lado de la familia, por lo que el centro educativo es un aspecto crítico para los individuos.

En investigaciones anteriores, Creed y colaboradores (2003) y Eccles y colaboradores (2004) sobre ajuste académico han demostrado que participar en el centro educativo tiene un impacto significativo en el bienestar psicológico de los estudiantes. Los estudiantes que se encuentran mejor ajustados académicamente reciben calificaciones más altas y se desempeñan mejor en las pruebas estandarizadas (Ladd \& Dinella, 2009, Schunk \& Pajares, 2005). También tienden a utilizar estrategias de logro funcional optimistas y centradas en la tarea (Mäkikangas \& Kinnunen, 2003, Salmela-Aro et al., 2009) y tienen menos probabilidades de sufrir depresión en la vida académica posterior (Salmela-Aro, et al., 2011).

Así mismo, se ha demostrado que los niveles reducidos de participación tienen un impacto grave en los estudiantes, lo que resulta en un bajo rendimiento, una mayor incidencia de conductas negativas y un mayor riesgo de abandonar la escuela (Finn \& Rock, 1997, Wu et al., 2010). 
Por otro lado, para contener la propagación de la pandemia COVID-19, muchos países instituyeron cierres temporales de instituciones de educación superior en marzo de 2020. Según la Organización de las Naciones Unidas para la Educación, la Ciencia y la Cultura (UNESCO, 2020a), las instituciones de educación superior estaban cerradas, lo que afectó aproximadamente 1.300 millones de estudiantes en todo el mundo. Como consecuencia, los estudiantes se han enfrentado a una situación fundamentalmente alterada no solo con respecto a sus estudios sino también con su vida en su conjunto, debido a las múltiples medidas de contención.

Los bloqueos, las limitaciones de movimiento, la interrupción de las rutinas, el distanciamiento físico, la restricción de las interacciones sociales y la privación de los métodos de aprendizaje tradicionales han provocado un aumento del estrés, ansiedad y preocupaciones sobre la salud mental de los estudiantes de todo el mundo (UNESCO, 2020b). En general, COVID-19 y sus medidas de contención han creado desafíos únicos para el bienestar psicológico. Para contrarrestar los resultados negativos del desarrollo, se deben identificar recursos que fomenten el bienestar en tiempos de crisis.

De acuerdo con lo expuesto, se buscó determinar la asociación del bienestar psicológico con el ajuste académico de los estudiantes de la universidad pública de Guatemala. La recolección de datos se hizo durante los meses de septiembre a noviembre del 2020 , mientras aún había algunas restricciones en el país y las clases se desarrollaban de forma virtual a causa de la COVID-19.

\section{Fundamentación teórica}

\section{Ajuste académico}

Se refiere a la participación en tareas y actividades relacionadas con la institución educativa (Appleton, et al., 2006). Dentro de la literatura, se percibe como un metaconstructo compuesto por dimensiones conductuales, cognitivas y emocionales (Archambault, et al., 2009, Fredericks, et al., 2004, Sharkey, et al., 2008 \& Zaff et al., 2011). 
El compromiso conductual implica la participación del estudiante en grupos sociales, interacción en el aula, estudio, tanto en el centro educativo como en el hogar, y actividades extracurriculares relacionadas con esta (Archambault, et al., 2009, Powell et al., 2008). El compromiso cognitivo, por otro lado, se refiere a la inversión personal del estudiante en las actividades de aprendizaje, incluida la autorregulación, el compromiso con el dominio del aprendizaje y el uso de estrategias de estudio (Sedeghat, et al., 2011). El compromiso emocional abarca los factores afectivos del ajuste académico, incluido el disfrute, el apoyo, la pertenencia y las actitudes hacia los maestros, los compañeros, el aprendizaje y la universidad en general (Eccles et al., 1997; Watt, 2004).

Se ha sugerido que los componentes emocionales, cognitivos y conductuales de la participación escolar capturan las dimensiones relacionadas pero que se desarrollan por separado que contribuyen a la participación del estudiante en la institución educativa (Jimerson, et al., 2003, Wang \& Eccles, 2011).

La investigación sobre el ajuste académico ha sido criticada por enfatizar la adaptación a las expectativas y normas establecidas en el contexto escolar (Chapman, 2003) al afirmar que la falta de comportamiento disruptivo (Caraway, et al., 2003) es uno de los criterios principales de participación en lugar de centrarse en una experiencia escolar significativa. Esto descuida el núcleo del ajuste académico, es decir, una experiencia escolar positiva y satisfactoria que promueve un aprendizaje significativo. Skinner y Belmont (1993) descubrieron que los estudiantes que participaban en actividades de aprendizaje mostraban una implicación conductual acompañada de un tono emocional positivo, un esfuerzo y concentración intensos en la tarea de aprendizaje. Sin embargo, sorprendentemente se sabe poco acerca de cómo el compromiso emocional construido en las relaciones con los compañeros y la interacción maestro-alumno contribuye al compromiso cognitivo del alumno, cómo estos ingredientes están interrelacionados y, además, cómo estos factores promueven la participación de los estudiantes en 
la realización de actividades de aprendizaje (Fall \& Roberst, 2012; Goodenow, 1993; Jennings, 2003; Skinner \& Belmont, 1993 \& Skinner, et al., 2009).

Además, el ajuste académico está estrechamente relacionada con el contexto social y las necesidades básicas del estudiante. Las relaciones sociales, el apoyo a la autonomía, la competencia para realizar las tareas en la universidad, la capacidad para actuar de manera autónoma en relación con las habilidades de autorregulación y el establecimiento de buenas relaciones con los demás son los determinantes de los aspectos cognitivo, emocional y conductual del compromiso escolar. También produce varios beneficios, que incluyen el rendimiento académico y la graduación, la conciencia social y la capacidad de establecer buenas relaciones con los demás, la conciencia emocional, la regulación de las emociones y las habilidades para la resolución de conflictos (Appleton, et al., 2008). Además, los estudios han revelado que el alto nivel de ajuste académico de los estudiantes está relacionado con un nivel reducido de conductas de riesgo, como fumar, uso de sustancias, depresión, pensamientos suicidas, agresión y experiencia sexual temprana y conduce a conductas saludables como hacer ejercicio, sexo seguro y usar casco al andar en bicicleta con más frecuencia (Allen et al. 2018 \& Carter et al. 2007).

\section{Bienestar psicológico}

Es multifacético e incluye aspectos tanto eudaimónicos (propósito, realización) como hedónicos (sentirse bien). Se ha demostrado que predice una amplia gama de resultados de salud, como una mejor salud subjetiva (Benyamini et al., 2000), menos afecciones crónicas (Friedman \& Ryff, 2012) y menores tasas de mortalidad (Boyle et al., 2009; Diener \& Chan, 2011).

Además, se trata de que la vida vaya bien. Es la combinación de sentirse bien y funcionar con eficacia. El bienestar sostenible no requiere que las personas se sientan bien todo el tiempo; la experiencia de emociones dolorosas como decepción, fracaso, 
dolor, son una parte normal de la vida, y ser capaz de manejar estas emociones negativas o dolorosas es esencial para el bienestar a largo plazo. Sin embargo, el bienestar psicológico se ve comprometido cuando las emociones negativas son extremas o muy duraderas e interfieren con la capacidad de una persona para funcionar en su vida diaria.

El interés por el estudio del bienestar ha aumentado dramáticamente dentro del campo de las ciencias sociales durante las últimas décadas. Busca evaluar cómo los individuos funcionan psicológicamente en respuesta a las demandas de su vida basándose en la noción eudaimónica de que el bienestar proviene de la realización del potencial humano (Ryan \& Deci, 2001). Se concibe como un constructo multidimensional compuesto por seis dimensiones: autoaceptación (ser consciente y aceptar las propias fortalezas y debilidades), relaciones positivas (tener relaciones profundas y significativas con los demás), crecimiento personal (experimentar el progreso como resultado del desarrollo y fortalezas de uno mismo), autonomía (ser autodeterminado y tomar decisiones independientes), dominio del entorno (manejar las circunstancias propias para aprovechar las oportunidades) y propósito en la vida (establecer metas y ser guiado por ellas). La conjunción de estas dimensiones resultó de la convergencia de diferentes modelos previos que intentaron describir la salud mental, como las teorías de Jahoda, (1958), Maslow (1968) o Rogers (1961). El objetivo principal era ofrecer una base basada en la teoría para evaluar los indicadores de funcionamiento positivo (Ryff, 1989; Ryff \& Keyes, 1995).

A su vez, estas dimensiones, y en particular el dominio ambiental, están estrechamente relacionadas con el sentido de autonomía y la capacidad de autodeterminación e independencia del individuo (Rosa-Rodríguez et al., 2015). Como resultado, estos indicadores a menudo se denominan "activos de salud" dado que afectan la salud física y mental de los jóvenes y, en última instancia, el desarrollo de su comportamiento (Chen, et al., 2019). 
Además, se ha determinado que correlatos sociodemográficos, como la edad, están vinculados al bienestar psicológico de diversas formas. También está relacionado con constructos psicológicos, como experiencias de vida, inteligencia emocional y rasgos de personalidad, y que existe una correlación positiva significativa en el ámbito educativo, en referencia a los aspectos personales. crecimiento y propósito en la vida (Bucchianeri, et al., 2016; Butler-Barnes et al., 2017 \& Henn et al., 2016).

\section{Materiales y métodos}

El estudio es de enfoque cuantitativo, no experimental de corte transversal y alcance correlacional predictivo, con un muestreo no probabilístico por voluntarios, quienes respondieron un cuestionario virtual alojado en la plataforma soscisurvey.de/. Participó en total 347 estudiantes de la universidad pública de Guatemala.

Para el ajuste académico se utilizó la Medida de Participación Escolar (Fredricks et al., 2004; Maroco et al, 2016), compuesta de 19 ítems con un tipo Likert de 5 puntos formato de respuesta ( $1=$ nunca y $5=$ todo el tiempo). El análisis factorial de los elementos de la versión original tiene las escalas, correspondientes al compromiso conductual (5 ítems), compromiso emocional (6 ítems) y compromiso cognitivo (8 ítems).

Para el bienestar psicológico se utilizó la adaptación española de Díaz y colaboradores (2006). Incluye 29 ítems que evalúan las 6 dimensiones propuestas por Ryff (1989): autoaceptación (4 ítems); autonomía (6 ítems); relaciones positivas con otras personas ( 5 ítems); crecimiento personal (4 ítems); dominio del entorno (5 ítems); y propósito en la vida (6 ítems). Comprende de 1 , en total desacuerdo y 5 totalmente de acuerdo. Por último, un cuestionario sociodemográfico el cual tenía dos secciones, datos académicos y personales. 
Los datos se analizaron en el programa de distribución libre Jamovi con su integración a $R$, versión 1.8.4. Se comenzó con las medidas de tendencia central, frecuencias y porcentajes de cada variable. Además, la prueba de confiabilidad Alfa de Crombach's; análisis exploratorio de los datos Kaiser-Mayer-Olkin (KMO) y su prueba de Bartlett.

En cuanto a la dicotomización de las variables se tomó como punto de corte la media del puntaje total de las respuestas de los evaluados.

La asociación fue a través de una regresión logística binomial. Se incluyó el modelo y la varianza ajustada (Nagelkerke $R^{2}$ ) de la dependiente. Además, para la hipótesis de la regresión se comprobó por medio de la prueba Hosmer Lemeshow.

Todos los datos recolectados tuvieron los procedimientos éticos adecuados. Se informó a todos los participantes por medio del consentimiento informado los principios éticos, riesgos, confidencialidad de los datos y justicia. 
Resultados y discusión

Tabla 1

\begin{tabular}{|c|c|c|}
\hline \multicolumn{3}{|l|}{ Datos sociodemográficos } \\
\hline Variables & $f$ & $\%$ \\
\hline \multicolumn{3}{|l|}{ Sexo } \\
\hline Hombre & 119 & $34.30 \%$ \\
\hline Mujer & 228 & $65.70 \%$ \\
\hline \multicolumn{3}{|l|}{ ¿Tiene empleo? } \\
\hline Si & 196 & $56.50 \%$ \\
\hline No & 151 & $43.50 \%$ \\
\hline \multicolumn{3}{|l|}{ Estado civil } \\
\hline Soltera(o) & 302 & $87 \%$ \\
\hline Unida(o) & 26 & $7.50 \%$ \\
\hline Casada(o) & 15 & $4.30 \%$ \\
\hline Separada(o) & 1 & $0.30 \%$ \\
\hline Divorciada(o) & 2 & $0.50 \%$ \\
\hline Viuda(o) & 1 & $0.30 \%$ \\
\hline \multicolumn{3}{|l|}{ Unidad Académica } \\
\hline Psicología & 121 & $34.90 \%$ \\
\hline Económicas & 96 & $27.70 \%$ \\
\hline Medicina & 9 & $2.60 \%$ \\
\hline Humanidades & 34 & $9.80 \%$ \\
\hline Arquitectura & 23 & $6.60 \%$ \\
\hline Diseño Gráfico & 38 & $11 \%$ \\
\hline Otra & 26 & $7.50 \%$ \\
\hline \multicolumn{3}{|l|}{ Jornada } \\
\hline Matutina & 94 & $27.10 \%$ \\
\hline Vespertina & 67 & $19.30 \%$ \\
\hline Nocturna & 170 & $49 \%$ \\
\hline Fin de Semana & 16 & $4.60 \%$ \\
\hline \multicolumn{3}{|l|}{ Nivel de profesión } \\
\hline $\begin{array}{l}\text { Carrera técnica o profesorado } \\
\text { (Pregrado) }\end{array}$ & 32 & $9.20 \%$ \\
\hline Licenciatura, ingeniería (Grado) & 310 & $89.30 \%$ \\
\hline $\begin{array}{l}\text { Postgrado (especialización, maestría, } \\
\text { doctorado) }\end{array}$ & 5 & $1.40 \%$ \\
\hline
\end{tabular}

$\mathrm{N}=347$

Fuente: Elaboración propia 
La edad media es de 22 ( $S D=2.09)$. La mayoría son mujeres (65.70\%), principalmente de la jornada nocturna (49\%). El resto de los datos están en la Tabla 1.

Tabla 2

Cruce de ajuste académico y bienestar psicológico

\begin{tabular}{cccccccc}
\hline $\begin{array}{l}\text { Bienestar } \\
\text { psicológico }\end{array}$ & \multicolumn{9}{c}{ Ajuste académico } & \multicolumn{2}{l}{ Total } \\
& \multicolumn{3}{c}{ Indicador bajo } & \multicolumn{2}{c}{ Indicador alto } \\
\multicolumn{1}{c}{$f$} & $\%$ & $f$ & $\%$ & $f$ & $\%$ & \\
Indicador bajo & 128 & $36.90 \%$ & 50 & $14.40 \%$ & 178 & $51.30 \%$ \\
Indicador alto & 61 & $17.60 \%$ & 108 & $31.10 \%$ & 169 & $48.70 \%$ \\
$\quad$ Total & 189 & $54.50 \%$ & 158 & $45.50 \%$ & 347 & $100 \%$ \\
$\mathrm{~N}=347$ & & & & & & & \\
\hline
\end{tabular}

Fuente: Elaboración propia

El ajuste académico tiene $M=70.60(S D=8.27) ; \alpha=.794$ y un $K M O=.842$, mientras que el bienestar psicológico $M=$ $126(S D=19.80) ; \alpha=.893$ y un $K M O=.90$. Ambas escalas fueron significativas en la prueba de Bartlett's $(p<.001)$. Los resultados dicotómicos se pueden observar en la Tabla 2.

\section{Tabla 3}

Análisis: bienestar psicológico y ajuste académico

Efecto

Estimado

SE

Bajo

Supe-

rior
Intercepto

Bienestar psicológicoa

$\mathrm{N}=347$

a0 = indicador bajo, 1 = indicador alto $\begin{array}{lllll}-0.571 & 0.16 & 0.413 & 0.773 & <.001\end{array}$

$\begin{array}{lllll}1.511 & 0.231 & 2.881 & 7.131 & <.001\end{array}$

Fuente: Elaboración propia 
En cuanto a la asociación de bienestar psicológico en ajuste académico, la regresión fue significativa $\times 2(2, N=347)=$ $45.80, p<.001$; Nagelkerke $R^{2}=16.50 \%$ de la varianza. Se clasificó correctamente $68.40 \%$. La especificidad es de 68.40 $\%$ y la sensibilidad $67.70 \%$. La razón de probabilidades (OR) para un indicador alto de bienestar psicológico es de 4.532, lo cual muestra que el bienestar psicológico predice el ajuste académico. La Tabla 3 detalla el resto de los resultados.

Para determinar si las variables tienen un ajuste adecuado se realiza la prueba de Hosmer Lemeshow que da como resultado $p=.064$, lo cual nos dice que se acepta el modelo planteado.

\section{Discusión}

El estudio evaluó el papel mediador del bienestar psicológico en la asociación entre el ajuste académico. De acuerdo con los resultados del análisis de regresión logística binomial, hubo relaciones positivas significativas $\left(r^{2}=1.511\right)$ entre el bienestar psicológico y ajuste académico, tal como se esperaba. Como resultado del modelo seleccionado, la variable independiente juega un papel mediador en la relación entre la variable dependiente. Este modelo respalda todos los roles de mediación entre los constructos. En otras palabras, un aumento en el ajuste escolar aumenta el bienestar psicológico.

En la literatura anterior y relacionada, hay investigaciones acerca de la relación entre las variables de estudio. Los estudios longitudinales han apoyado que el ajuste académico se asocia significativamente con el bienestar psicológico de los estudiantes. En grados menores a la universidad, con altos indicadores de ajuste académico, más tarde informaron una mayor satisfacción escolar y efectos positivos más frecuentes en la institución académica (Tian, et al., 2016). Otro estudio mostró una gran correlación positiva con el bienestar psicológico, mientras que hubo una relación moderadamente negativa entre la pertenencia a la escuela y la angustia 
emocional (Arslan 2018). Los alumnos con indicadores más altos de ajuste académico experimentaron emociones más positivas y disminución en las negativas, lo que contribuyó a su rendimiento escolar (Lam, et al., 2015).

En cuanto al compromiso escolar cognitivo, emocional y conductual del universitario tiene varios resultados positivos, incluido el rendimiento académico, el bienestar emocional y social (Appleton, et al., 2008 \& Archambault, et al., 2009). El compromiso emocional en las relaciones entre maestros, estudiantes y grupos de pares había predicho el bienestar experimentado en la universidad (Pietarinen et al. 2014). En un estudio longitudinal que monitoreó a adolescentes, se encontró que existe una relación positiva entre bienestar psicológico, que predice mejores indicadores de compromiso emocional. La naturaleza del bienestar psicológico orientado a un propósito es necesario para el desarrollo y mantenimiento del bienestar en los adolescentes (Ciarrochi, et al., 2015). Los autores You y colaboradores (2008) encontraron que este aumenta la satisfacción con la vida de los estudiantes a través del compromiso escolar. A medida que aumenta en los alumnos, también aumentan el ajuste académico y la satisfacción con la vida. Los estudiantes con altos indicadores de bienestar psicológico también tienen altos niveles de competencia social (Snyder, et al., 1997). Se acepta también como un determinante de la cohesión psicológica y social (Snyder, 2002). Estas investigaciones apoyan los roles mediadores entre las variables propuestas.

Según Konu y Rimpela (2002), los altos indicadores de bienestar psicológico se refieren a una situación escolar saludable que facilita el comportamiento positivo de los estudiantes en la institución educativa. Una universidad saludable puede conducir a la felicidad y crear una actitud y creencias positivas de modo que, indirectamente, afecte a los estudiantes cuando interactúan con ella. 
Uno de los factores que afectan el bienestar psicológico de los estudiantes es el compromiso cognitivo, emocional y conductual, que los lleva a tener una mejor salud mental. En Guatemala, los intentos de establecerla en las instituciones educativas no ha sido la agenda principal; este asunto todavía se considera responsabilidad de los psicólogos.

Por otra parte, el bienestar psicológico llevaría a una situación escolar que permite a las personas satisfacer sus necesidades básicas, tanto materiales como no materiales (Konu \& Rimpela, 2002). Esta situación abarcaría un aspecto material y no material como el entorno físico, la asignatura y el horario, el castigo y el servicio en el centro educativo.

Es así como el bienestar psicológico es una de las consecuencias del desarrollo de la psicología positiva dentro de la universidad. Es una condición generalizada que la vida presente y futura sea placentera, una percepción continua de que la vida es significativa y satisfactoria (O'Brien, 2008). Además, es necesario porque la institución educativa tiene un efecto significativo en el desarrollo de los alumnos y en ella pasan la mayor parte de su tiempo, de cinco a seis días a la semana. También afecta positivamente el proceso y el resultado del aprendizaje. Quienes estén satisfechos en el centro educativo desarrollarán actitudes positivas hacia el proceso y el logro del aprendizaje. Así mismo, permite desarrollar una estrategia de afrontamiento contra la influencia ambiental negativa (Jarvela, 2011).

Según Wang y Hoocombe (2010) quienes poseen indicadores altos de bienestar psicológico y ajuste académico tienden a involucrarse en su universidad, transformarán su energía en actividades positivas, dedicarán su tiempo a desarrollar su potencial académico y a impulsar su motivación en participar activamente en actividades dentro o fuera del aula. Quienes participan en el proceso de aprendizaje mejorarán su capacidad, competencia y valores que les ayudarán a pasar correctamente su etapa de transición. 
En forma contraria puede aumentar la posibilidad de que los estudiantes se depriman, se sientan incapaces o incluso creen un problema emocional y de comportamiento. Esto, por supuesto, dificulta el proceso de aprendizaje. En consecuencia, la variables son factores importantes que afectan el desempeño de los alumnos en la educación superior.

Por otro lado, se considera que el modelo planteado es significativo y el tamaño de la muestra como fortalezas sustanciales de la investigación. Los resultados están respaldados por los procedimientos estadísticos adecuados, lo que indica que la misma ecuación de la regresión y los mismos significados atribuidos a los constructos latentes pueden asumirse en los centros universitarios donde se hizo la recopilación de datos.

A pesar de estas notables fortalezas, la presente investigación es limitada en algunos aspectos. Los datos se recopilaron en línea. Esto llevó a una autoselección de nuestra muestra por voluntarios. Se debe tener en claro que se buscó tener un adecuado poder estadístico dentro de los análisis planteados. Además, debido a la novedad de la situación del COVID-19, algunas de nuestras medidas fueron adaptadas para este estudio. Sin embargo, se tomaron todas las medidas adecuadas para tener instrumentos con confiabilidad y validez pertinentes, lo cual se presenta en el apartado de resultados.

\section{Conclusiones}

El paradigma de la psicología positiva no solo afecta a muchas áreas de la psicología, sino que también en la práctica de la psicología educativa. Hay muchas ventajas en centrarse en las características positivas de los estudiantes. Fredrickson (2013) sostiene que el bienestar psicológico tiene muchos beneficios tanto a corto como a largo plazo. Los estudios sugieren que los psicológicos en las instituciones educativas deberían realizar estudios para desarrollar constructos relacionados al bienestar (Howell, et al., 2013; Terjesen, et al., 2004). Los programas de psicología positiva pueden 
aumentar el bienestar psicológico y el ajuste académico de los universitarios (Seligman, et al. 2009). Las intervenciones que se realizarán para garantizar el impacto positivo con estas variables deben incluir estudios para desarrollar habilidades de participación social como el apoyo social (José, et al., 2012). Actividades positivas, como la satisfacción de las necesidades básicas, el desarrollo de rasgos de carácter positivos como la esperanza, afrontamiento y el compromiso y la conexión con la naturaleza, mejoraran la salud mental (Howell et al., 2013). Estudiantes con altos niveles de bienestar tienen bajos niveles de depresión y problemas de comportamiento y altos niveles de comportamientos prosociales y compromiso con el centro educativo (Keyes, 2013).

Con base a los resultados de este estudio, podemos sugerir que, para aumentar el bienestar psicológico de los estudiantes, se deben realizar programas de intervención para desarrollar su compromiso escolar cognitivo, emocional y conductual junto con actividades que aumenten la resiliencia y aseguren que exhiban comportamientos prosociales. Los psicólogos que buscan un mejor desarrollo de los universitarios pueden hacer uso de estos hallazgos al planificar la consejería individual y grupal, y programas psicoeducativos, así como estudios para mejorar el bienestar y las culturas escolares positivas.

Comprender los factores más fuertemente asociados con el ajuste académico puede dar pistas sobre dónde deberían producirse los cambios en el sistema. Los resultados del estudio actual pueden contribuir a la literatura de psicología educativa y psicología positiva sobre la comprensión de los constructos en ámbitos universitarios. 


\section{Referencias}

Allen, K., Kern, M. L., Vella-Brodrick, D., Hattie, J., \& Waters, L. (2018). What schools need to know about fostering school belonging: A meta-analysis. Educational

Psychology Review, 30(1), 1-34. https://doi.org/10.1007/ s10648-016-9389-8.

Appleton, J. J., Christenson, S. L., Kim, D., \& Reschly, A.

L. (2006). Measuring cognitive and psychological engagement: Validation of the Student Engagement

Instrument. Journal of school psychology, 44(5), 427445. https://doi.org/10.1016/j.jsp.2006.04.002

Appleton, J. J., Christenson, S. L., \& Furlong, M. J. (2008). Student engagement with school: Critical conceptual and methodological issues of the construct. Psychology in the Schools, 45, 369-386. https://doi.org/10.1002/ pits.20303.

Archambault, I., Janosz, M., Fallu, J. S., \& Pagani, L. S. (2009). Student engagement and its relationship with early high school dropout. Journal of adolescence, 32(3), 651-670. https://doi.org/10.1016/j.adolescence.2008.06.007

Arslan, G. (2018). Exploring the association between school belonging and emotional health among adolescents. International Journal of Educational Psychology, 7(1), 21-41. https://doi.org/10.17583/ijep.2018.3117

Benyamini, Y., Idler, E. L., Leventhal, H., \& Leventhal, E. A. (2000). Positive affect and function as influences on self-assessments of health: expanding our view beyond illness and disability. The journals of gerontology. Series $B$, Psychological sciences and social sciences, 55(2), P107-P116. https://doi.org/10.1093/geronb/55.2.p107

Boyle, P. A., Barnes, L. L., Buchman, A. S., \& Bennett, D. A. (2009). Purpose in life is associated with mortality among community-dwelling older persons. Psychosomatic medicine, 71(5), 574-579. https://doi.org/10.1097/ PSY.0b013e3181a5a7c0 
Bucchianeri, M. M., Fernandes, N., Loth, K., Hannan, P. J., Eisenberg, M. E., \& Neumark-Sztainer, D. (2016). Body dissatisfaction: Do associations with disordered eating and psychological well-being differ across race/ethnicity in adolescent girls and boys?. Cultural diversity \& ethnic minority psychology, 22(1), 137-146. https://doi. org/10.1037/cdp0000036

Butler-Barnes, S. T., Martin, P. P., \& Boyd, D. T. (2017). African American adolescents' psychological well-being: The impact of parents' religious socialization on adolescents' religiosity. Race and Social Problems, 9(2), 115-126. https:// doi.org/10.1007/s12552-017-9199-8

Caraway, K., Tucker, C. M., Reinke, W. M., \& Hall, C. (2003). Self- efficacy, goal orientation, and fear of failure as predictors of school engagement in high school students. Psychology in the Schools, 40(4), 417-427. https://doi. org/10.1002/pits.10092

Carter, M., McGee, R., Taylor, B., \& Williams, S. (2007). Health outcomes in adolescence: Associations with family, friends and school engagement. Journal of Adolescence, 30(1), 5162. https://doi.org/10.1016/j.adolescence.2005.04.002.

Chapman, E. (2003). Alternative approaches to assessing student engagement rates. Practical assessment, research \& evaluation, 8(13), 1-10.

Chen, Y., Kim, E. S., Koh, H. K., Frazier, A. L., \& VanderWeele, T. J. (2019). Sense of mission and subsequent health and well-being among young adults: an outcome-wide analysis. American journal of epidemiology, 188(4), 664673. https://doi.org/10.1093/aje/kwz009

Ciarrochi, J., Parker, P., Kashdan, T. B., Heaven, P. C., \& Barkus, E. (2015). Hope and emotional well-being: A sixyear study to distinguish antecedents, correlates, and consequences. The Journal of Positive Psychology, 10(6), 520-532. https://doi.org/10.1080/17439760.2015.101 5154 
Creed, P. A., Muller, J., \& Patton, W. (2003). Leaving high school:

The influence and consequences for psychological well-being and career-related confidence. Journal of adolescence, 26(3), 295-311. https://doi.org/10.1016/ S0140-1971(03)00015-0

Díaz, D., Rodríguez-Carvajal, R., Blanco, A., Moreno-Jiménez, B., Gallardo, I., Valle, C., \& Van Dierendonck, D. (2006). Adaptación española de las escalas de bienestar psicológico de Ryff. Psicothema, 18(3), 572-577.

Diener, E., \& Chan, M. Y. (2011). Happy people live longer:

Subjective well- being contributes to health and longevity. Applied Psychology: Health and Well- Being, 3(1), 1-43. https://doi.org/10.1111/j.1758-0854.2010.01045.x

Eccles, J. S., Vida, M. N., \& Barber, B. (2004). The relation of early adolescents' college plans and both academic ability and task-value beliefs to subsequent college enrollment. The Journal of Early Adolescence, 24(1), 6377. https://doi.org/10.1177/0272431603260919

Eccles, J. S., Midgley, C., Wigfield, A., Buchanan, C. M., Reuman, D., Flanagan, C., \& Mac Iver, D. (1997). Development during adolescence: The impact of stage-environment fit on young adolescents' experiences in schools and in families (1993). In J. M. Notterman (Ed.), The evolution of psychology: Fifty years of the American Psychologist (pp. 475-501). American Psychological Association. https:// doi.org/10.1037/10254-034

Fall, A. M., \& Roberts, G. (2012). High school dropouts: Interactions between social context, self-perceptions, school engagement, and student dropout. Journal of adolescence, 35(4), 787-798. https://doi.org/10.1016/j. adolescence.2011.11.004

Finn, J. D., \& Rock, D. A. (1997). Academic success among students at risk for school failure. Journal of Applied Psychology, 82(2), 221-234. https://doi. org/10.1037/0021-9010.82.2.221 
Fredricks, J. A., Blumenfeld, P. C., \& Paris, A. H. (2004). School engagement: Potential of the concept, state of the evidence. Review of educational research, 74(1), 59-109. https://doi.org/10.3102/00346543074001059

Fredrickson, B. L. (2013). Positive emotions broaden and build. In Advances in experimental social psychology (Vol. 47, pp. 1-53). Academic Press.

Friedman, E. M., \& Ryff, C. D. (2012). Living well with medical comorbidities: a biopsychosocial perspective. The journals of gerontology. Series B, Psychological sciences and social sciences, 67(5), 535-544. https://doi. org/10.1093/geronb/gbr152

Goodenow, C. (1993). Classroom belonging among early adolescent students: Relationships to motivation and achievement. The journal of early adolescence, 13(1), 2143. https://doi.org/10.1177/0272431693013001002

Henn, C. M., Hill, C., \& Jorgensen, L. I. (2016). An investigation into the factor structure of the Ryff Scales of Psychological Well-Being. SA Journal of Industrial Psychology, 42(1), 1-12. http://dx.doi.org/10.4102/sajip. v42i 1.1275

Howell, A. J., Keyes, C. L., \& Passmore, H. A. (2013). Flourishing among children and adolescents: Structure and correlates of positive mental health, and interventions for its enhancement. In Research, Applications, and Interventions for Children and Adolescents (pp. 59-79). Springer Netherlands.

Jahoda, M. (1958). Current concepts of positive mental health. Basic Books. https://doi.org/10.1037/11258-000

Jarvela, S. (Ed.). (2011). Social and emotional aspects of learning. Elsevier.

Jennings, G. (2003). An exploration of meaningful participation and caring relationships as contexts for school engagement. The California School Psychologist, 8(1), 43-51. https://doi.org/10.1007/BF03340895 
Jimerson, S. R., Campos, E., \& Greif, J. L. (2003). Toward an understanding of definitions and measures of school engagement and related terms. The California School Psychologist, 8(1), 7-27. https://doi.org/10.1007/ BF03340893

Jose, P. E., Ryan, N., \& Pryor, J. (2012). Does social connectedness promote a greater sense of well-being in adolescence over time? Journal of Research on Adolescence, 22(2), 235-251. https://doi.org/10.1111/ j.1532-7795.2012.00783.x

Keyes, C. L. (2013). Promoting and protecting positive mental health: Early and often throughout the lifespan. In Mental well-being (pp. 3-28). Springer Netherlands.

Konu, A., \& Rimpelä, M. (2002). Well-being in schools: a conceptual model. Health promotion international, 17(1), 79-87. https://doi.org/10.1093/heapro/17.1.79

Ladd, G. W., \& Dinella, L. M. (2009). Continuity and change in early school engagement: Predictive of children's achievement trajectories from first to eighth grade? Journal of Educational Psychology, 101(1), 190206. https://doi.org/10.1037/a0013153

Lam, U. F., Chen, W. W., Zhang, J., \& Liang, T. (2015). It feels good to learn where I belong: School belonging, academic emotions, and academic achievement in adolescents. School Psychology International, 36(4), 393-409. https:// doi.org/10.1177/0143034315589649

Mäkikangas, A., \& Kinnunen, U. (2003). Psychosocial work stressors and well-being: Self-esteem and optimism as moderators in a one-year longitudinal sample. Personality and individual differences, 35(3), 537-557. https://doi.org/10.1016/S0191-8869(02)00217-9

Maroco, J., Maroco, A., Campos, J., \& Fredricks, J. (2016). University student's engagement: development of the University Student Engagement Inventory (USEI). Psicologia: Reflexão e Crítica, 29(1), 1-12. https://doi. org/10.1186/s41155-016-0042-8 
Maslow, A. (1968). Some educational implications of the humanistic psychologies. Harvard Educational Review, 38(4), 685-696. https://doi.org/10.17763/ haer.38.4.j07288786v86w660

Pietarinen, J., Soini, T., \& Pyhältö, K. (2014). Students' emotional and cognitive engagement as the determinants of well-being and achievement in school. International Journal of Educational Research, 67, 40-51. https://doi.org/10.1016/j.ijer.2014.05.001

Powell, D. R., Burchinal, M. R., File, N., \& Kontos, S. (2008). An eco-behavioral analysis of children's engagement in urban public school preschool classrooms. Early Childhood Research Quarterly, 23(1), 108-123. https:// doi.org/10.1016/j.ecresq.2007.04.001

O'Brien, M. (2008). Well-being and post-primary schooling. NCCA.

Organización de las Naciones Unidas para la Educación, la Ciencia y la Cultura (2020a). COVID-19 impact on education. https://en.unesco.org/covid19/ educationresponse

Organización de las Naciones Unidas para la Educación, la Ciencia y la Cultura (2020b). Nurturing the social and emotional wellbeing of children and young people during crises. UNESCO COVID-19 Education Response. https://unesdoc.unesco.org/ark:/48223/ pf0000373271

Rogers, C. R. (1995). On becoming a person: A therapist's view of psychotherapy. Houghton Mifflin Harcourt.

Rosa-Rodríguez, Y., Negrón Cartagena, N., Maldonado Peña, Y., Quiñones Berrios, A., \& Toledo Osorio, N. (2015). Dimensiones de bienestar psicológico y apoyo social percibido con relación al sexo y nivel de estudio en universitarios. Avances en psicología latinoamericana, 33(1), 33-43. 
Ryan, R. M., \& Deci, E. L. (2000). Self-determination theory and the facilitation of intrinsic motivation, social development, and well-being. The American psychologist, 55(1), 68-78. https://doi.org/10.1037//0003-066x.55.1.68

Ryff, C. D. (1989). Happiness is everything, or is it? Explorations on the meaning of psychological well-being. Journal of Personality and Social Psychology, 57(6), 10691081. https://doi.org/10.1037/0022-3514.57.6.1069

Ryff, C. D., \& Keyes, C. L. M. (1995). The structure of psychological well-being revisited. Journal of Personality and Social Psychology, 69(4), 719-727. https://doi. org/10.1037/0022-3514.69.4.719

Salmela-Aro, K., Kiuru, N., Leskinen, E., \& Nurmi, J. E. (2009). School burnout inventory (SBI) reliability and validity. European journal of psychological assessment, 25(1), 48-57. https://doi.org/10.1027/1015-5759.25.1.48

Salmela-Aro, K., Tolvanen, A., \& Nurmi, J. E. (2011). Social strategies during university studies predict early career work burnout and engagement: 18-year longitudinal study. Journal of Vocational Behavior, 79(1), 145-157. https://doi.org/10.1016/j.jvb.2011.01.002

Santrock, J. W. (2009). Psicologia educacional. AMGH Editora.

Schunk, D. H., \& Pajares, F. (2005). Competence perceptions and academic functioning. In A. J. Elliot \& C. S. Dweck (Eds.), Handbook of competence and motivation (pp. 85104). Guilford Press

Sedaghat, M., Abedin, A., Hejazi, E., \& Hassanabadi, H. (2011). Motivation, cognitive engagement, and academic achievement. Procedia-Social and Behavioral Sciences, 15, 2406-2410. https://doi.org/10.1016/j. sbspro.2011.04.117

Seligman, M. E., Ernst, R. M., Gillham, J., Reivich, K., \& Linkins, M. (2009). Positive education: Positive psychology and classroom interventions. Oxford Review of Education, 35(3), 293-311. https://doi. org/10.1080/03054980902934563 
Seligman, M. E. (2012). Flourish: A visionary new understanding of happiness and well-being. Simon and Schuster.

Sharkey, J. D., You, S., \& Schnoebelen, K. (2008). Relations among school assets, individual resilience, and student engagement for youth grouped by level of family functioning. Psychology in the Schools, 45(5), 402-418. https://doi.org/10.1002/pits.20305

Skinner, E. A., \& Belmont, M. J. (1993). Motivation in the classroom: Reciprocal effects of teacher behavior and student engagement across the school year. Journal of Educational Psychology, 85(4), 571-581. https://doi. org/10.1037/0022-0663.85.4.571

Skinner, E.A., Kindermann, T.A.,Connell, J.P.,\& Wellborn, J.G.(2009). Engagement as an organizational construct in the dynamics of motivational development. In K. Wentzel \& A. Wigfield (Eds.), Handbook of motivation in school (pp. 223-245). Erlbaum.

Snyder, C. R., Hoza, B., Pelham, W. E., Rapoff, M., Ware, L., Danovsky, M., Highberger, L., Rubinstein, H., \& Stahl, K. J. (1997). The development and validation of the Children's Hope Scale. Journal of pediatric psychology, 22(3), 399-421. https://doi.org/10.1093/ jpepsy/22.3.399

Snyder, C. R., Feldman, D. B., Shorey, H. S., \& Rand, K. L. (2002). Hopeful choices: A school counselor's guide to hope theory. Professional School Counseling, 5(5), 298307.

Tian, L., Zhang, L., Huebner, E. S., Zheng, X., \& Liu, W. (2016). The longitudinal relationship between school belonging and subjective well-being in school among elementary school students. Applied Research in Quality of Life, 11(4), 1269-1285. https://doi.org/10.1007/s11482015-9436-5. 
Terjesen, M. D., Jacofsky, M., Froh, J., \& DiGiuseppe, R. (2004). Integrating positive psychology into schools: Implications for practice. Psychology in the Schools, 41(1), 163-172. https://doi.org/10.1002/pits.10148

Wang, M. T., \& Eccles, J. S. (2012). Adolescent behavioral, emotional, and cognitive engagement trajectories in school and their differential relations to educational success. Journal of research on adolescence, 22(1), 3139. https://doi.org/10.1111/j.1532-7795.2011.00753.x

Wang, M. T., \& Holcombe, R. (2010). Adolescents' perceptions of school environment, engagement, and academic achievement in middle school. American educational research journal, 47(3), 633-662. https://doi. org/10.3102/0002831209361209

Watt, D. (2004). Consciousness, emotional self-regulation and the brain. Journal of Consciousness Studies, 11(9), 7782.

You, S., Furlong, M. J., Felix, E., Sharkey, J. D., Green, J. G., \& Tanigawa, D. (2008). Relations among school connectedness, hope, life satisfaction, and bully victimization. Psychology in the Schools, 45, 446-460. https://doi.org/10.1002/pits.20308

Zaff, J. F., Kawashima-Ginsberg, K., Lin, E. S., Lamb, M., Balsano, A., \& Lerner, R. M. (2011). Developmental trajectories of civic engagement across adolescence: Disaggregation of an integrated construct. Journal of Adolescence, 34(6), 1207-1220. https://doi. org/10.1016/j.adolescence.2011.07.005 


Semblanza académica del autor
Licenciado en psicología, especialidad en Psicología
Social por la Escuela de Ciencias Psicológicas y Msc.
en Educación Superior de la Facultad de Humanidades,
ambos en la Universidad San Carlos de Guatemala
(USAC). Corrector de estilo profesional y científico por
la Universidad Autónoma de México. Entre la experiencia
profesional esta ser profesor investigador en la Unidad
Profesional de Investigación en la Escuela de Ciencias
Psicológicas de la USAC. Coordinador, investigador
y auxiliar II de investigación en la Dirección General
de Investigación (DIGI-USAC). Así mismo, consultor
independiente para Organizaciones no Gubernamentales.
Autor de capítulos de libros científicos y artículos en
revistas indexadas.

